\title{
Parallelimporte patentgeschützter Güter schaden dem Forschungsplatz Schweiz
}

\author{
D. Grauer*
}

\begin{abstract}
In der Diskussion um die Kosten im Gesundheitswesen wird immer wieder die Zulassung von Parallelimporten gefordert - in der Hoffnung, damit die Arzneimittelpreise und infolgedessen die Kosten der obligatorischen Krankenversicherung zu senken. Notwendig ist indessen eine differenzierte Sichtweise, denn namentlich die Zulassung von Parallelimporten patentgeschützter Arzneimittel wäre geeignet, den Forschungsplatz Schweiz nachhaltig zu schädigen.
\end{abstract}

\footnotetext{
* Stellvertretender Direktor der Schweizerischen Gesellschaft für Chemische Industrie (SGCI), Zürich, www.sgci.ch.
}

1 Urteil des Bundesgerichts vom 7. Dezember 1999 in Sachen Kodak SA gegen Jumbo Markt AG (BGE 126 III 129).

2 In der Herbstsession 2002 lehnte es der Nationalrat ab, der Revision des Patentgesetzes vorzugreifen und im Kartellgesetz eine Bestimmung vorzusehen, die den Patentschutz in bezug auf die Zulassung von Parallelimporten relativierte.

3 Urteil des Bundesgerichts vom 23. Oktober 1996 in Sachen Chanel S.A. Genève et Chanel S.A. gegen EPA AG (BGE 122 III 469).

4 Urteil des Bundesgerichts vom 20. Juli 1998 in Sachen Imprafot AG gegen Nintendo Co. Ltd. und Waldmeier AG.

5 Bei den Arzneimitteln gilt für die wirtschaftlich nutzbare Patentschutzdauer eine Sonderregelung. Da bei den Arzneimitteln Prüfungs- und Zulassungsverfahren vorgeschrieben sind, die 10 bis 12 Jahre beanspruchen, kann die gesetzliche Normalschutzdauer nicht ausgenützt werden. Für Arzneimittel kann der Staat daher ergänzende Schutzzertifikate für maximal fünf Jahre erteilen.

Korrespondenz:

Dr. iur. Dieter Grauer

Schweizerische Gesellschaft

für Chemische Industrie (SGCI)

Nordstrasse 15

CH-8035 Zürich
Es mag überraschen, ist aber eine Tatsache: Parallelimporte in die Schweiz sind keineswegs generell verboten. Sie sind im Gegenteil in den weitaus meisten Fällen möglich und können den Wettbewerb beleben. Doch was sind Parallelimporte überhaupt und weshalb kommt es dazu? Der Preis identischer und immaterialgüterrechtlich geschützter Waren kann von Land zu Land verschieden hoch sein. Die Gründe dafür sind vielfältig: So führen beispielsweise Unterschiede in der Kaufkraft oder bei den Lebensgewohnheiten zu Unterschieden beim Angebot oder bei der Nachfrage solcher Produkte. Bei Arzneimitteln sind dabei staatliche Preiseingriffe, wie sie in vielen Ländern üblich sind, von besonderer Bedeutung. Überschreiten die Preisunterschiede zwischen den Ländern ein gewisses Ausmass, so entstehen wirtschaftliche Anreize, diese auszunutzen (sogenannte Arbitrage): Händler beschaffen sich die Ware in einem Niedrigpreisland, importieren sie in ein Hochpreisland und verkaufen sie dort mit einem Preisvorteil. Diesen Vorgang nennt man einen Parallelimport. Die Parallelimporteure konkurrieren damit die Herstellerin im Hochpreisland oder die Handelsfirma, die dieses Produkt dort vermarktet.

\section{Parallelimporte und Schutzrechte}

In der Politik wird darüber gestritten, ob der Parallelimport aufgrund der Schutzrechte, die sich aus dem geistigen Eigentum ergeben, zu Recht verunmöglicht werde. So beabsichtigt die nationalrätliche Kommission für Wirtschaft und Abgaben (WAK), die Diskussion um die Zulassung von Parallelimporten bei der laufenden Teilrevision des Kartellgesetzes (KG) wieder aufzunehmen. Die WAK weicht damit vom Antrag des Bundesrates ab. Dieser klammerte in seiner Botschaft zur KG-Revision die Frage der Parallelimporte ausdrücklich aus. Er hielt sich dabei an die vom Bundesgericht im Kodak-Urteil [1] vertretene Auffassung, wonach das Kartellrecht anwendbar sei, wenn der Patentinhaber, vereinfacht gesagt, seine ihm mit dem Patent eingeräumte Monopolstellung missbrauche [2]. In diesem Kontext ist auf die Tatsache hinzuweisen, dass in der Schweiz nur die patentrechtlich geschützten Güter vom Parallelimport ausgenommen sind - mit gutem Grund, wie noch auszuführen ist. In der Schweiz errichten, wie das Bundesgericht entschieden hat, das Marken- [3] und das Urheberrecht [4] keine Schutzmauern gegen Parallelimporte. Ein kurzer Blick auf die Eigenheiten dieser Schutzrechte im Zusammenhang mit der Parallelimportfrage macht dies deutlich:

\section{Markenschutz}

Die Marke soll Waren oder Dienstleistungen eines Unternehmens von solchen anderer Unternehmen unterscheiden und die Konsumenten vor Täuschung und Verwechslung schützen. Das Schweizer Markenrecht lässt - anders als etwa dasjenige der Europäischen Union (EU) den Parallelimport zu, besteht doch für den Konsumenten beim Parallelimport keine Gefahr der Täuschung oder Verwechslung. Der Markeninhaber kann sein Schutzrecht regelmässig erneuern und somit seinen Schutz zeitlich unbeschränkt geltend machen. Werden in der Schweiz Parallelimporte markengeschützter Produkte mit Alleinvertriebsverträgen ausgeschlossen, so greifen die Bestimmungen des Kartellrechts. Es ist dann an der Wettbewerbskommission (WEKO) aufgrund einer Güterabwägung zu beurteilen, ob Verträge, mit denen der Hersteller oder seine Vertriebsfirma Parallelimporte verhindern wollen, mit dem Kartellrecht vereinbar sind.

\section{Patentschutz}

Das Patentrecht soll die technische Innovation im Interesse der Allgemeinheit fördern. Mit der Erteilung des Patentes kommt es zu einem Interessenausgleich. Der Staat schützt eine Erfindung für eine zeitlich beschränkte Dauer von 20 Jahren [5]. Er verleiht dem Patentinhaber das Recht, 
andere von der gewerbsmässigen Nutzung seiner Erfindung auszuschliessen. Damit schafft das Patent den wirtschaftlichen Anreiz, auf dem Gebiet der Technik wertschöpfende Leistungen zu erbringen. Im Gegenzug muss der Erfinder seine Erfindung mit der Patentschrift offenlegen, also für jedermann öffentlich zugänglich machen. Mit der Gewährung des gesetzlich befristeten Monopols hindert der Staat Trittbrettfahrer daran, eine Erfindung zu nutzen, ohne dass sie sich an den Forschung- und Entwicklungskosten beteiligen. Dieser Aufwand kann sehr hoch sein. Für ein neues Arzneimittel ist heute bis zu einer Milliarde Franken oder mehr einzusetzen. Der Erfinder soll eine faire Chance haben, zumindest seinen Aufwand zu erwirtschaften. Diese Chance darf nicht durch Parallelimporte zunichte gemacht werden. Dieses Prinzip ist weltweiter Standard. Nur gerade Argentinien und Hongkong lassen Parallelimporte patentgeschützter Güter zu.

\section{Urheberschutz}

Mehr der Vollständigkeit halber sei der Urheberschutz erwähnt. Das Urheberrecht regelt den Schutz der Urheber von Werken der Literatur und Kunst, der ausübenden Künstler, der Hersteller von Ton- und Tonbildträgern sowie der Sendeunternehmen. Es fördert somit das kreative Schaffen vor allem im kulturellen Bereich. Analog zum Patentrecht gewährt das Urheberrecht einen zeitlich limitierten Schutz. Dieser erlischt aber erst 70 Jahre nach dem Tod des Urhebers (bei Computerprogrammen 50 Jahre nach dem Tod des Urhebers). Dem Urheber bzw. seinen Rechtsnachfolgern verschafft der Urheberschutz somit eine sehr lange Verwertungsdauer. Die Schweiz erlaubt analog zum Markenrecht Parallelimporte urheberrechtlich geschützter Güter.

\section{Bekenntnis zum Forschungsplatz Schweiz nötig}

Marken- und Urheberrecht sind also kein Schutzwall gegen Parallelimporte. Ausschliesslich das Patentrecht verwehrt Parallelimporte geschützter Produkte. Das seit Beginn dieses Jahres geltende Heilmittelgesetz sieht die Zulassung parallelimportierter Arzneimittel nur vor, wenn deren Patentschutz abgelaufen ist. Wer nun fordert, der Patentschutz solle als Parallelimportschranke fallen, verkennt die mit diesem Schutz verfolgten innovationsfördernden und volkswirtschaftlichen Ziele der staatlichen Rechtsordnung.
Der Staat will dem Erfindungsschutz als Anreiz für Forschung und Entwicklung den Vorrang einräumen - vor einem schrankenlosen Wettbewerb, der diese aufgrund einer Güterabwägung als übergeordnet bestimmten Ziele verfehlte. Ohne Patente wäre die Forschung mit dem Ziel, Arzneimittel zur Bekämpfung heute noch nicht heil- oder behandelbarer Krankheiten zu finden, ernstlich in Frage gestellt. Aber auch die Forschung mit dem Ziel, therapeutisch bessere, sicherere (vor allem nebenwirkungsärmere) und möglichst patientenfreundlich anwendbare Arzneimittel zu entwickeln, könnte kaum fortgesetzt werden. Ohne Patentschutz würden neue Arzneimittel durch Nachahmer umgehend kopiert; dem Erfinder bliebe nichts ausser seine hohen Forschungs- und Entwicklungskosten. Die Zulassung von Parallelimporten patentgeschützter Produkte unterliefe den für die Innovation so wichtigen Patentschutz. Sie schmälerte damit nicht nur die Innovationsprämie des einzelnen Erfinders, sondern schädigte auch den gesamten Forschungs- und Werkplatz Schweiz. Als rohstoffarmes und forschungsstarkes Land hat die Schweiz alles Interesse, Parallelimporte patentgeschützter Produkte auch weiterhin nicht zuzulassen. Gäbe sie diese Position auf, so gewährte sie allen WTO-Mitgliedländer eine Konzession - und zwar ohne jedes Gegenrecht. Auch eine auf die Arzneimittel beschränkte Sonderregelung wäre nicht möglich, da eine nach Technologien unterschiedliche Regelung des Parallelimports dem WTO-Recht ebenso widerspräche wie eine unterschiedliche Regelung nach Ländern oder Ländergruppen.

\section{Gerechtfertigte Unterschiede zwischen Marken-, Urheber- und Patentschutz}

Sinnvoll und gerechtfertigt sind dagegen die in der Schweiz heute bestehenden Unterschiede zwischen dem Marken- und Urheberschutz einerseits (der Parallelimporte erlaubt) und dem Patentschutz andererseits (der Parallelimporte untersagt):

- Marken- und Urheberrecht gewähren einen zeitlich unbeschränkten bzw. sehr langen Schutz und somit auch eine lange Verwertungszeit. Dem Patentinhaber hingegen steht nur eine vergleichsweise wesentlich kürzere Dauer zur Verfügung, um seine Investitionen in die Forschung und Entwicklung seines Produktes zu amortisieren. 
- Parallelimporte haben keinen Einfluss auf die Kennzeichnungs- und Herkunftsfunktion der Marke. Auch die Urheberrechte werden nicht tangiert. Hingegen vermindern Parallelimporte patentgeschützter Produkte den Anreiz zu forschen und zu entwickeln.

Oft wird geltend gemacht, was bezüglich Parallelimporte in der EU möglich sei, sollte auch der Schweiz nicht verwehrt sein. Ein direkter Vergleich geht allerdings fehl: Die EU ist eine Zollunion, hat einen gemeinsamen Binnenmarkt und versteht sich als wirtschaftliche Einheit. Aufgrund der Warenverkehrsfreiheit sind Parallelimporte innerhalb der EU zugelassen. Parallelimporte aus Drittstaaten verweigert sie aber nicht nur bei geltendem Patentschutz, sondern auch bei marken- und urheberrechtlich geschützten Produkten. Die EU ist somit beim Marken- und Urheberrecht weniger offen als

\section{Kein gesetzgeberischer Handlungsbedarf}

Allfälligen Missbräuchen des befristeten Monopols, welches das Patentrecht einräumt, kann wettbewerbsrechtlich begegnet werden, wie das Bundesgericht im Kodak-Urteil unterstrichen hat. Das bestehende Instrumentarium dazu genügt; einer Kartellgesetzänderung bedarf es dafür nicht. Der volkswirtschaftliche Schaden, der unserem Land mit einem Aufbruch der patentrechtlichen Schranke gegen Parallelimporte brächte, lässt sich derzeit noch nicht beziffern. Hierzu sollten die vom Bundesrat aufgrund von parlamentarischen Vorstössen bestellten Berichte noch dieses Jahr Aufschluss geben. Bekannt ist hingegen nach den Erfahrungen sowie aufgrund der von der EU-Kommission in Auftrag gegebenen sogenannten NERA-Studie [6], dass mit der Zulassung der Parallelimporte im EU-Raum zur Hauptsache die Parallelimporteure profitieren und die Konsumenten kaum etwas davon spüren. 